\title{
Soft Decoding Assisted Interference Cancellation in a Non-binary LDPC Coded Fast Frequency Hopping Multiuser System Using Product Combining
}

\author{
Sohail Ahmed, Lie-Liang Yang, Lajos Hanzo and Feng Guo \\ School of ECS, University of Southampton, SO17 1BJ, UK. \\ Tel: +44-23-8059 3364, Fax: +44-23-8059 4508 \\ Email: \{sa03r,lly,lh\}@ecs.soton.ac.uk; http://www-mobile.ecs.soton.ac.uk
}

\begin{abstract}
In this contribution, soft-input-soft-output (SISO) decoding aided successive interference cancellation (SIC) is considered in fast frequency hopping (FFH), $M$-ary frequency shift keying (MFSK) using product combining (PC), when operating in multiple access (MA) Nakagami- $m$ channels. All users' $M$ ary source symbols are encoded using non-binary LDPC codes and the encoded symbols are transmitted using FFH-MFSK modulation. A sub-optimum soft metric is proposed which is based on self-normalization of the diversity combiner outputs. In the context of the proposed SIC scheme, the receiver exploits the soft information fed back by the LDPC decoder to the demodulator in order to cancel the interference imposed by the reliably detected symbols. Our simulation results show that the proposed scheme is capable of combatting the effects of multiuser interference and outperforms conventional iterative decoding by about $1 \mathrm{~dB}$.
\end{abstract}

\section{INTRODUCTION}

In fast frequency hopping (FFH), $M$-ary frequency shift keying (MFSK) systems operating in a multiple access (MA) scenario [1], multiuser interference (MUI) is the main source of performance degradation [1]. Given the knowledge of all users' addresses, the base station (BS) receiver may invoke multiuser detection (MUD) [2] by jointly detecting all users' signals to combat MUI. Specifically, when a multi-stage successive interference cancellation (SIC) assisted MUD [1] is employed, the effects of symbols reliably detected at a specific SIC stage can be removed in the subsequent stages, thereby reducing the interference constituted by these reliably detected users. In our previous work on this topic [3], we introduced a novel MUD scheme that invokes SIC in conjunction with SISO decoding. In [3], we employed binary convolutional encoded FFH-MFSK using clipped combining.

Against this background, the novel contribution of this paper is that we extend the idea of SISO assisted SIC to product combining $(P C)$ [4] and employ non-binary LDPC codes [5], [6] instead of binary convolutional encoding, which creates a purely symbol-based $M$-ary scheme. More explicitly, the $M$-ary symbols are encoded using LDPC codes over Galois Field (GF) $(q)$. Furthermore, by employing non-binary LDPC

The financial support of the EPSRC, UK and EU under the auspices of the Phoenix and Newcom projects and of Higher Education Commission, Pakistan is gratefully acknowledged. encoding, we can process symbol probabilities in the reciever, instead of log-likelihood ratios (LLRs) which allows us to dispense with converting from symbol probabilities to LLRs and vice versa. Moreover, since a FFH-MFSK modulated symbol is transmitted in multiple hops using a different frequency in each hop duration, the channel appears uncorrelated to the transmitted signal and therefore no interleaving of the symbols is needed. Hence, the proposed system is eminently suitable for low-delay applications. Furthermore, we consider Nakagami$m$ fading channels in this contribution, since this allows us to investigate the system's performance over more generalized fading. Finally, since it is challenging to derive the soft metrics for the PC receiver, we propose a sub-optimum metric for deriving the required symbol probabilities from the diversity combiner's outputs. We will compare the performance of the proposed SIC scheme with that invoking single user detection (SUD) and conventional iterative decoding (ID) between the SISO decoder and the FFH-MFSK demodulator.

The rest of this paper is structured as follows. In Sec. II, the system under consideration is described and in Sec. III, the soft metrics are derived and the proposed SIC scheme is discussed. In Sec. IV, we will demonstrate with the aid of our simulation results that the proposed SIC method results in useful performance gains. Finally, in Sec. V, we present our conclusions.

\section{SYSTEM DESCRIPTION}

The FFH-MFSK system considered consists of a single cell, serving multiple users with the aid of a BS and utilizing a spread spectrum bandwidth of $W_{s s}$. The schematic of the proposed system is depicted in Fig. 1. All users' $M$-ary symbols are encoded by a nonbinary LDPC code [5], as shown in Fig. 1. The encoded $M$-ary symbols are modulated using a FFH-MFSK modulator as follows. The $k$ th user, $k=1,2, \ldots, N_{U}$, is assigned a unique and random $L$-tuple FFH address $\mathbf{a}_{\mathbf{k}}=\left[a_{k}(0), a_{k}(1), \ldots, a_{k}(L-1)\right]$, which is output by the PN generator seen in Fig. 1, where $a_{k}(l) \in$ $\mathrm{GF}(M), l=0,1, \ldots, L-1$, and $L$ is the number of frequency hops per symbol. In the MFSK modulator of Fig. 1, an MFSK tone is chosen for transmission whose frequency during the $l$ th hop of duration $T_{h}$ is chosen according to [1]

$$
\mathcal{S}_{\mathbf{k}}=\left[\mathcal{S}_{k}(0), \mathcal{S}_{k}(1), \ldots, \mathcal{S}_{k}(L-1)\right]=X_{k} . \mathbf{1} \oplus \mathbf{a}_{\mathbf{k}},
$$




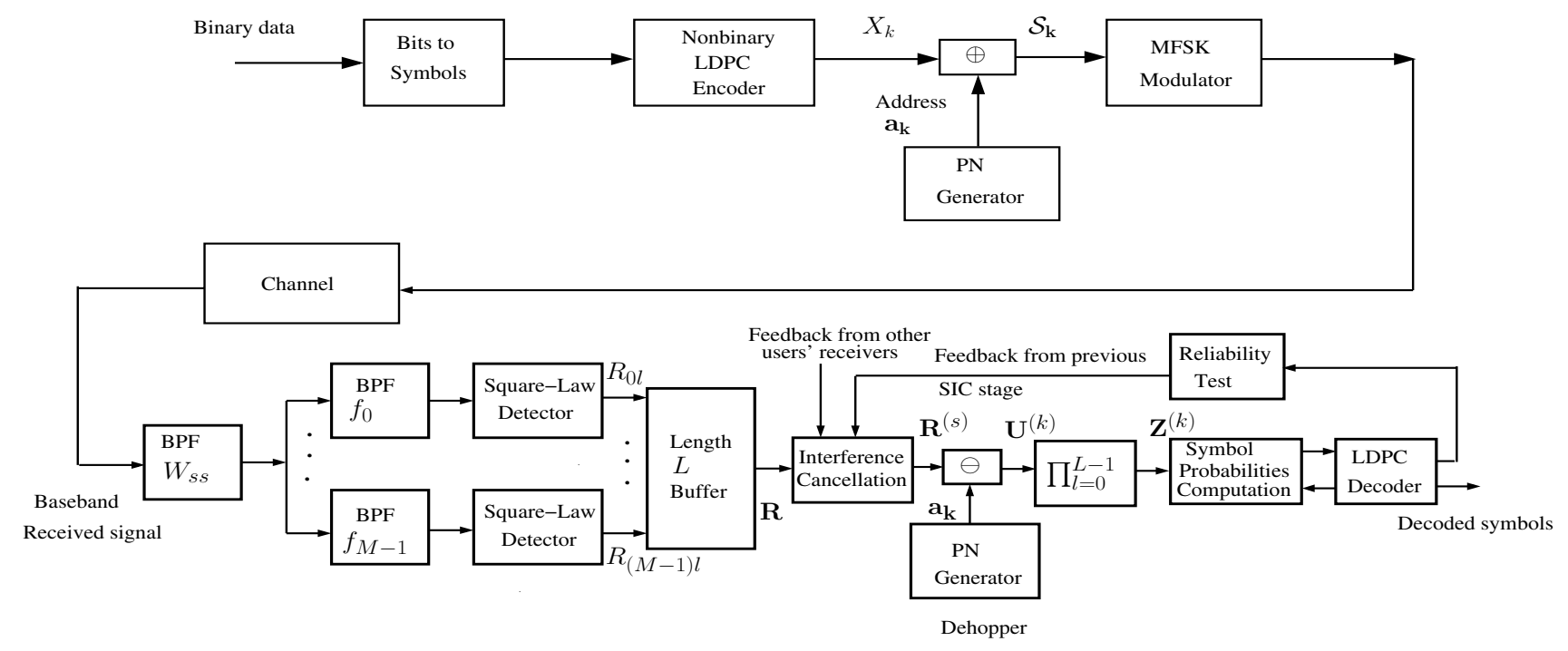

Fig. 1. Block diagram of the FFH-MFSK system for the $k$ th user, $k=1,2, \ldots, N_{U}$, employing non-binary LDPC channel coding and SIC in the receiver.

where 1 denotes a unit vector of length $L, X_{k}$ is the $M$ ary symbol to be transmitted by the $k$ th user and $\oplus$ denotes addition in the GF. Note that $\mathcal{S}_{k}(l), a_{k}, X_{k} \in[0,1, \ldots, M-$ 1]. We assume that the bandwidth occupied by the signal transmitted during each FFH chip interval is given by $R_{h}=$ $1 / T_{h}$. The frequency separation between adjacent FH tones is also assumed to be $R_{h}$. Thus, the orthogonality of the FFH frequency tones is maintained. Note that for ease of analysis, random FFH patterns are assumed [1].

The channel is modeled by uncorrelated, frequency-flat Nakagami- $m$ fading for each transmitted frequency. We assume that the frequency separation between the adjacent signalling frequencies is higher than the coherence bandwidth of the channel. Thus each signalling tone can be assumed to experience independent fading. The fading envelopes experienced by the various users are also independent of each other. Furthermore, the transmitted signals are corrupted by additive white Gaussian noise (AWGN) having a one-sided power spectral density of $N_{0}$.

When a particular user transmits a signal to the BS, the signals transmitted by all the other users may be modeled as continuous-wave interfering tones. For the sake of simplicity, we also assume a chip-synchronous system, where the chip transitions of all users are aligned in time. Furthermore, perfect power control is assumed, hence the power of all the signals received by the BS from all users is identical.

The BS receiver's schematic is also shown in Fig. 1, where we assume that the hopping sequence of the FFH demodulator is in synchronism with that of the FFH modulator of the transmitter. After carrier demodulation, a bank of $M$ squarelaw detectors detect the energy received in each MFSK tone. The outputs of the detectors are dehopped and combined over $L$ hops using PC, as will be discussed in the next section. Then soft decoding and SIC is employed for cancelling the MUI, which is also discussed in the context of the proposed
SIC scheme in the next section.

\section{SOFt Information BASED SIC SCHEME}

As seen in Fig. 1, the decoder accepts soft inputs from the demodulator, employs SISO decoding and delivers soft $a$ posteriori information as well as the decoded symbols. In the following section we discuss how soft information is derived by the demodulator from the channel observations.

\section{A. Suboptimum Soft Metric}

A number of suboptimum soft metrics have been proposed for the family of noncoherent MFSK based systems [7]-[9]. Most of these metrics derive the LLR values from the detectors' outputs. In order to employ a soft metric for our proposed SIC scheme, it should be based on the symbol probabilities rather than on the LLRs, because as we will discuss in Sec. III$\mathrm{B}$, our test of symbols are based on the symbol probabilities. Hence, we invoke a soft metric which is derived by selfnormalization of the diversity combiners' outputs. This soft metric was also discussed in [9], [10], although it has not been employed for the PC receiver before. In the context of this metric, the a posteriori symbol probability corresponding to the $n$th PC output of the $k$ th user is given by

$$
P_{M, a p o}^{(k)}(n)=\frac{Z_{n}^{(k)}}{\sum_{j=0}^{M-1} Z_{j}^{(k)}}, \quad n=0,1, \ldots, M-1,
$$

where $\mathbf{Z}^{(k)}=\left[Z_{0}^{(k)}, Z_{1}^{(k)}, \ldots, Z_{M-1}^{(k)}\right]$ represents the set of $M$ PC outputs for the $k$ th user, seen in Fig. 1. Furthermore, in (2), the subscript $(M$, apo $)$ denotes the a posteriori symbol probability corresponding to the MFSK based demodulator.

The non-binary LDPC decoder accepts the set of $M$ symbol probabilities $\mathbf{P}_{M, \text { apo }}^{(k)}=$ $\left[P_{M, a p o}^{(k)}(0), P_{M, a p o}^{(k)}(1), \ldots, P_{M, a p o}^{(k)}(M-1)\right]$ from the demodulator and outputs a posteriori symbol probabilities 
$\mathbf{P}_{D, \text { apo }}^{(k)}$, which may be fed back to the demodulator as a priori symbol probabilities, for further assisting the demodulator to improve its soft information output. In conventional ID process, this exchange of soft information between the demodulator and decoder may be invoked a number of times [11].

\section{B. SIC Algorithm}

Let us now outline our proposed SIC algorithm. The joint SIC and SISO decoding process may be described as follows:

1) As seen in Fig. 1, the $M \times L$ order matrix $\mathbf{R}$, whose element $R_{n l}$ denotes the square-law detector output coresponding to the $n$th MFSK tone, $n=0,1, \ldots, M-$ 1 , in the $l$ th hop, $l=0,1, \ldots, L-1$, is separately dehopped by each receiver using the corresponding unique user address, resulting in the matrix $\mathbf{U}^{(k)}, k=$ $1,2, \ldots, N_{U}$, for the $k$ th user. The dehopping process may be expressed as

$$
\begin{aligned}
& U_{p l}^{(k)}=R_{n l}, \text { given } p=n \ominus a_{k}(l), \\
& n, p=0,1, \ldots, M-1 ; l=0,1, \ldots, L-1 .
\end{aligned}
$$

2) PC is performed on the dehopped signals of Fig. 1, resulting in $M$ decision variables for each of the users. The decision variable recorded after PC for the $k$ th user is given by [4]

$$
Z_{n}^{(k)}=\prod_{l=0}^{L-1} U_{n l}^{(k)}, \quad n=0,1, \ldots, M-1 .
$$

3) From the PC outputs of Fig. 1, the set of $M$ symbols probabilities $\mathbf{P}_{M \text {,apo }}^{(k)}$, derived using (2), are passed as $a$ priori input to the SISO decoder. The LDPC decoder and the demodulator may exchange extrinsic symbol probabilities with each other, the extrinsic output of one being used as the a priori input for the other. This process may be repeated $N_{I D}$ number of times, at the end of which the LDPC decoder generates the $a$ posteriori symbols probabilities $\mathbf{P}_{D, \text { apo }}^{(k)}$.

4) Next, as seen in Fig. 1 a reliability test of all users' symbols is carried out. Specifically, if the ratio of the second largest to the largest of the $M$ number of a posteriori probabilites $\mathbf{P}_{D, \text { apo }}^{(k)}$ corresponding to a symbol is less than a predefined threshold $\epsilon_{T}$, that is, if we have

$$
\epsilon^{(k)}=\frac{\max _{2}\left[\mathbf{P}_{D, \text { apo }}^{(k)}\right]}{\max \left[\mathbf{P}_{D, \text { apo }}^{(k)}\right]}<\epsilon_{T},
$$

then the symbol is declared reliable, else it is deemed unreliable. The reliable symbols are estimated from the a posteriori symbol probabilities $\mathbf{P}_{D, \text { apo }}^{(k)}$ using conventional hard decisions. Specifically, if the $k$ th user's symbol, $k=1,2, \ldots, N_{U}$ satisfies the reliability test given in (5), then $X_{k(d)}$ is the estimate of the $k$ th user's symbol, where $X_{k(d)}=0,1, \ldots, M-1$, is the index of the maximum of $\mathbf{P}_{D, a p o}^{(k)}$. If no user passes the test, we earmark that specific user's symbol for subsequent erasure, which has the lowest value of $\epsilon$.

5) If some but not all symbols are reliably detected, we proceed with the $s$ th stage of SIC, where $s=1,2, \ldots$. We also define $\mathbf{R}^{(s)}$ as the modified form of $\mathbf{R}$ at the $s$ th stage, as seen in Fig. 1. The sth stage of the SIC, $s \geq 1$, consists of the following steps:

a) The matrix $\mathbf{R}^{(s-1)}$ is modified by partial erasure of the elements representing the signals, which correspond to all the symbols that were declared reliable in the $(s-1)$ th stage (Step 4). More specifically, if $X_{k(d)}$ is the estimated symbol of the $k$ th user, then, after interference cancellation, we have

$$
R_{n l}^{(s)}= \begin{cases}\delta R_{n l}^{(s)} & \text { for } n=X_{k(d)} \oplus a_{k}(l) \\ R_{n l}^{(s-1)} & \text { otherwise, }\end{cases}
$$

where $0<\delta<1$ represents a suitably chosen cancellation coefficient.

b) Steps 1 to 4 outlined above are repeated for the matrix $\mathbf{R}^{(s)}$, to determine if more symbols can be declared reliable. In each SIC iteration, for symbols already declared reliable, we use the symbol probabilities $\mathbf{P}_{M, a p o}^{(k)}, k=1, \ldots, N_{U}$ obtained in the specific SIC stage in which they were declared reliable. For the remaining unreliable symbols, new values of symbol probabilities will be obtained after each iteration. Also note that we have $N_{I D}=$ 0 in each of the $s$ th SIC iteration, where $s \geq 1$.

6) After all symbols have satisfied the reliability test, or alternatively, when $N_{S I C}$ number of SIC iterations have been completed, we perform LDPC hard decoding using the symbol probabilities $\mathbf{P}_{M, \text { apo }}^{(k)}$ obtained at the end of last SIC stage.

We refer to this SIC scheme as the $\operatorname{SIC}\left(N_{I D}, N_{S I C}\right)$, to highlight the number of ID and SIC iterations invoked.

The reason for employing a cancellation coefficient $\delta$ in (6) is as follows. Suppose that we have $\delta=0$ in the above SIC scheme, implying a scenario where the signals corresponding to the reliable symbols are completely cancelled. It can be observed from our discussion of the SIC scheme that complete cancellation of the symbols which were declared reliable in a specific stage may lead to inadvertent cancellation of the hitherto undetected desired symbols, if two users happen to transmit the same FFH tone. This unintentional cancellation might lead to erroneous decisions and to subsequent propagation of decision errors, especially in the PC receiver where multiplication of the signals is employed. In order to avoid the resultant propagation of errors, we assume having a nonzero value of $\delta$, i.e. we have $0<\delta<1$, so that the signal is only partially cancelled in the next SIC stage and hence the potentially unwanted cancellations are avoided. Note also that $\delta=1$ implies no cancellation which corresponds to conventional detection. It has been demonstrated through our simulation results not included here for reasons of space economy that 
$\delta=0.3$ yields the best compromise in terms of bit error ratio (BER) results for most values of system parameters. In [12] a similar technique of partial cancellation was proposed, which employed a cancellation coefficient that was adapted based on the value of the fading parameter corresponding to a specific hop and MFSK tone. This technique would require the pilotassisted estimation of the fading envelope [12] and therefore may become overly complicated for practical implementation. Hence, we modify the partial cancellation mechanism of [12] by employing a constant cancellation coefficient seen in (6), thus creating a low-complexity SIC scheme that may be invoked in conjunction with PC.

A drawback of the proposed scheme is that the optimum test threshold $\epsilon$ defined in (5) has to be determined, which may depend upon the signal to noise ratio (SNR), the number of users, as well as the modulation and diversity order. However, all these parameters are known at the receiver. Hence the optimum test threshold $\epsilon$ may be determined using a search methodology.

\section{Simulation Results and Discussion}

In this section we present our BER performance results for the SIC scheme discussed in Sec. III, when employed in a FFH-MFSK system communicating over a frequency-flat Nakagami- $m$ fading MA channel. Comparison has been made with the SUD system employing conventional ID but no SIC. We have invoked LDPC code constructed over GF(16) and consequently results are shown for $M=16$ only. We employ a half-rate LDPC code having a block length of 24000 coded bits, i.e. 6000 4-bit symbols. Additionally, the optimum test thresholds $\epsilon$, defined in Sec. III, have been employed in all of our simulations. Note that we represent the number of internal iterations of the LDPC decoder as $N_{I N}$ and the number of iterations between the decoder and the demodulator in the context of conventional ID as $N_{I D}$, while the number of SIC iterations is denoted as $N_{S I C}$.

We commence with the $\operatorname{SIC}\left(0, N_{S I C}\right)$ configuration to highlight the effect of SIC iterations only. Fig. 2 shows the BER versus SNR performance of the SIC and SUD schemes, assuming $L=7$ and $N_{U}=8$ as well as Rayleigh fading associated with Nakagami paremeter of $m=1$. As a benchmarker, the single user (interference-free) performance of the FFH-MFSK scheme with $N_{I N}=4$ LDPC and $N_{I D}=1$ outer iteration is also included. The results of Fig. 2 demonstrate that at our target BER of $10^{-5}$, the SIC scheme yields approximately $3 \mathrm{~dB}$ gain compared to the SUD system using no outer iterations, and about $1 \mathrm{~dB}$ gain over that using $N_{I D}=2$ ID iterations. We also note that while the conventional ID process results in no iterative gain after $N_{I D}=1$ ID iteration, the SIC scheme yields BER improvements even in the $N_{S I C}=3$ iterations. In the subsequent discussions, unless otherwise specified, we employ $N_{S I C}=3 \mathrm{SIC}$ iterations and $N_{I D}=1$ ID iteration of the SUD scheme, since negligible iterative gain is achieved after these iterations, as evidenced in Fig. 2. Note that the performance of the proposed scheme is about $4 \mathrm{~dB}$ inferior to that of the single user scenario.

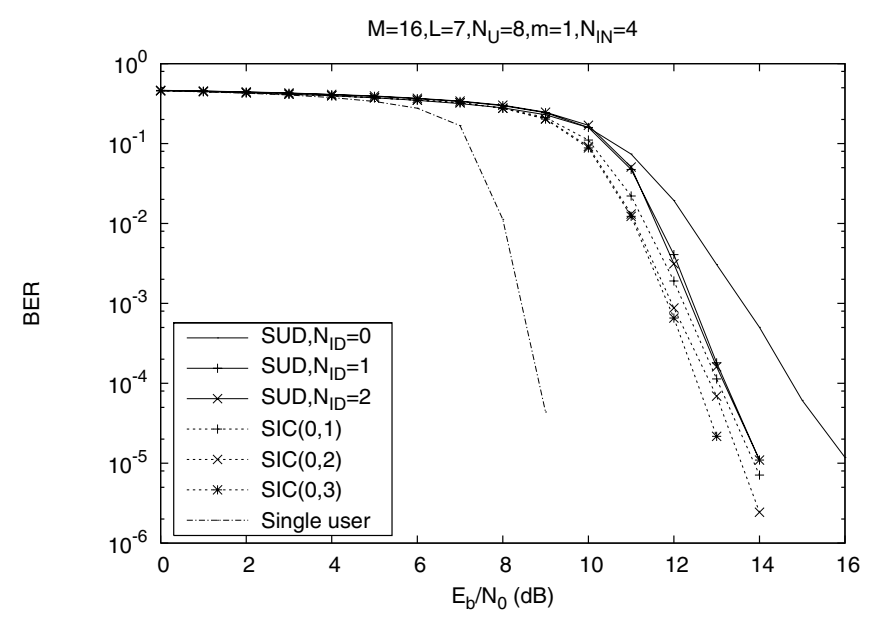

Fig. 2. BER versus the $E_{b} / N_{0}$ performance of non-binary LDPC coded FFH 16-ary FSK PC receiver communicating over a Rayleigh fading channel, assuming various number of ID and SIC iterations.

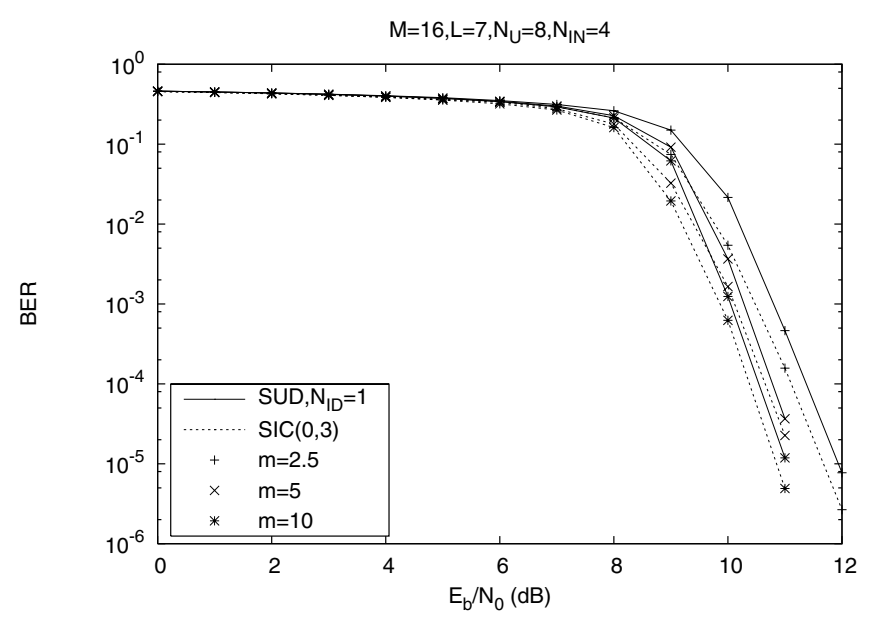

Fig. 3. BER versus the $E_{b} / N_{0}$ performance of non-binary LDPC coded FFH 16 -ary FSK PC receiver communicating over a Nakagami- $m$ fading channel, assuming various $m$ values.

In Fig. 3, we consider the BER versus the SNR performance of the two schemes considered for various values of the Nakagami parameter $m$. As expected, upon increasing $m$, the performance of both systems improves, although the performance difference between the SUD and the SIC scheme is reduced at higher $m$ values.

In Fig. 4, we consider the BER versus number of users performance of the schemes considered, where we assume $L=5$, Nakagami parameters of $m=1$ and 10 as well as $E_{b} / N_{0}=15 \mathrm{~dB}$. We observe that the SIC scheme performs significantly better than the SUD scheme in terms of supporting more users at a given BER value. Hence, at the BER values below $10^{-3}$, the SIC scheme is capable of supporting approximately $40 \%$ more users than the SUD scheme without ID iterations and nearly $25 \%$ more users than the SUD scheme 


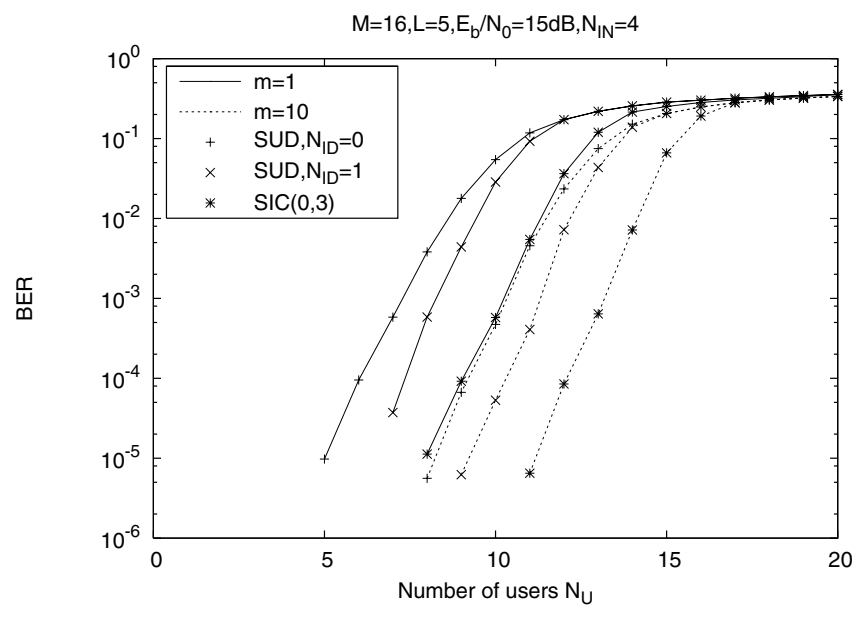

Fig. 4. BER versus number of users performance of non-binary LDPC coded FFH 16-ary FSK PC receiver communicating over a Nakagami- $m$ fading channel, assuming various $m$ values.

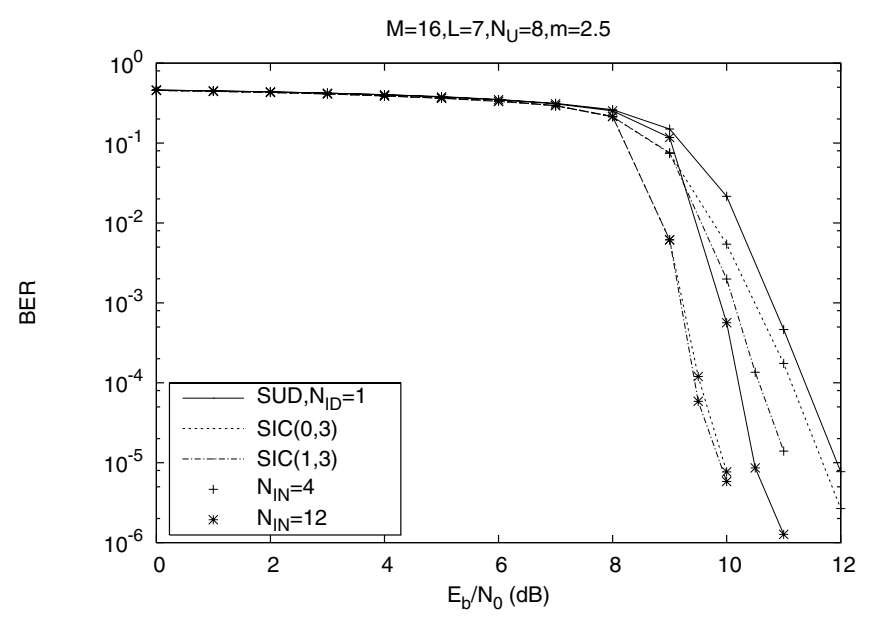

Fig. 5. BER versus the $E_{b} / N_{0}$ performance of non-binary LDPC coded FFH 16 -ary FSK PC receiver communicating over a Nakagami- $m$ fading channel, assuming various LDPC iterations.

invoking $N_{I D}=1$ ID iteration.

Finally, in Fig. 5, we show the BER versus SNR performance of the SIC $(1,3)$ configuration in comparison with those of the other configurations considered. It can be seen that the $\operatorname{SIC}(1,3)$ outperforms the $\operatorname{SIC}(0,3)$ configuration. We conclude that, assisted by $N_{I D}=1$ ID iteration, the SIC scheme is further improved and the $\operatorname{SIC}(1,3)$ yields a gain of nearly $1 \mathrm{~dB}$ over the SUD scheme and of about $0.5 \mathrm{~dB}$ over the $\operatorname{SIC}(0,3)$ arrangement at BER of $10^{-5}$. However, we also note that when $N_{I N}=12 \mathrm{LDPC}$ iterations are employed, the difference between the $\operatorname{SIC}(0,3)$ and the $\operatorname{SIC}(1,3)$ arrangements is not significant. The reason for this is that by employing a higher number of LDPC's inner iterations, the decoder's $a$ posteriori output is sufficiently improved, thereby rendering the additional ID iteration nearly insignificant.

\section{CONCLUSION}

We have investigated a SISO assisted SIC scheme designed for a FFH-MFSK receiver invoking PC and nonbinary LDPC decoding. We employed a sub-optimum method for deriving the symbol probabilities associated with the PC outputs. The proposed MUD scheme invokes SIC based on the feedback provided by the SISO decoder, and it is suitable for the PC receiver, since it invokes partial cancellation of the reliable symbols. Our results shown in Figs. 2 to 5 demonstrate that the proposed SIC scheme is capable of substantially enhancing the achievable performance of the FFH-MFSK receiver. Our SIC scheme outperforms the conventional SUD scheme and yields iterative gain in up to three SIC iterations, while the conventional SUD scheme yields no further BER improvement after the first ID iteration. An advantage of the proposed system is that no symbol interleaving is necessary, rendering the system eminently suitable for low-latency applications such as voice transmission.

\section{REFERENCES}

[1] U. C. Fiebig, "Iterative interference cancellation for FFH/MFSK MA systems," in IEEE Proceedings of International Conference on Communications, pp. 90-95, December 1996.

[2] L. Hanzo, L.-L. Yang, E.-L. Kuan, and K. Yen, Single- and Multi-Carrier DS-CDMA: Multi-User Detection, Space-Time Spreading, Synchronisation, Networking and Standards. New York: John Wiley and Sons, England, 2003

[3] S. Ahmed, S. X. Ng, L. L. Yang, and L. Hanzo, "Iterative Decoding and Soft Interference Cancellation in Fast Frequency Hopping Multiuser System Using Clipped Combining," in Proceedings of the IEEE Wireless Communications and Networking Conference, WCNC 2007 (CD-ROM)., March 2007.

[4] T. A. Gulliver, E. B. Felstead, R. E. Ezers, and J. S. Wright, "A unified approach to time diversity combining of fast frequency hopped NCMFSK - anti-jam processing," in IEEE Military Communications Conference, MILCOM '94, vol. 2, pp. 415-420, October 1994.

[5] F. Guo and L. Hanzo, "Low complexity non-binary LDPC and modulation schemes communicating over MIMO channels," in Proceedings of IEEE Vehicular Technology Conference, 2004, VTC2004-Fall, vol. 2, pp. 1294 - 1298, September 2004.

[6] A. Bennatan and D. Burshtein, "Design and analysis of nonbinary LDPC codes for arbitrary discrete-memoryless channels," IEEE Transactions on Information Theory, vol. 52, pp. 549 - 583, February 2006.

[7] Y. T. Su and R. C. Chang, "Performance of fast FH/MFSK Signals in jammed binary channels," IEEE Transactions on Communications, vol. 42, pp. $2414-2422$, July 1994.

[8] B. M. Peric, M. R. Souryal, E. Larsson, and B. R. Vojcic, "Soft Decision Metrics for Turbo-coded FH M-FSK Ad Hoc Packet Radio Networks," in IEEE 61st Vehicular Technology Conference, Spring 2005, vol. 2, pp. 724 - 727, May-June 2005.

[9] Y. Kim and K. Cheun, "Performance of soft metrics for convolutional coded asynchronous fast FHSS-MA networks using BFSK under Rayleigh fading," IEEE Transactions on Communications, vol. 51, pp. 5 -7 , January 2003.

[10] K. Cheun and W. E. Stark, "Performance of robust metrics with convolutional coding and diversity in FHSS systems under partial-band noise jamming," IEEE Transactions on Communications, vol. 41, pp. 200 209, January 1993.

[11] L. Hanzo, T. H. Liew, and B. L. Yeap, Turbo Coding, Turbo Equalisation and Space-Time Coding for Transmission over Fading Channels. England: John Wiley and Sons, England, 2002.

[12] K. Hamaguchi, L. L. Yang, and L. Hanzo, "On the performance of multistage multi-user detection assisted fast-FH/MFSK," in Proceedings of IEEE Vehicular Technology Conference, 2003, VTC2003-Spring, vol. 4, pp. 2798 - 2802, April 2003. 